\title{
SMC1 promotes epithelial-mesenchymal transition in triple-negative breast cancer through upregulating Brachyury
}

\author{
KAICHUN LI ${ }^{1}$, MINGZHEN YING $^{1}$, DAN FENG $^{1}$, YAN CHEN $^{2}$, JINGWEN WANG $^{3}$ and YAJIE WANG ${ }^{1}$ \\ ${ }^{1}$ Department of Oncology, Changhai Hospital Affiliated to The Second Military Medical University, Shanghai 200433; \\ Departments of ${ }^{2}$ Pathology and ${ }^{3}$ Oncology, Huadong Hospital Affiliated to Fudan University, Shanghai 200040, P.R. China
}

Received July 17, 2015; Accepted September 4, 2015

DOI: $10.3892 /$ or.2016.4564

\begin{abstract}
Triple-negative breast cancer (TNBC) is a special subtype of breast cancer, which is characterized by the negative form of estrogen receptor (ER), progesterone receptor (PR) and human epithelial growth factor receptor 2 (HER2). TNBC accounts for $\sim 15 \%$ of all breast cancer forms, and often leads to high mortality and poor prognosis. Structural maintenance of chromosome 1 (SMC1) is a subunit of the cohesion protein complex. Brachyury is a protein that is encoded by the $\mathrm{T}$ gene in humans, which is a transcription factor within the T-box complex of genes. Epithelial-mesenchymal transition (EMT) is a ubiquitous process in the body, and in particular, induces metastasis and the proliferation of cancer cells. In the present study, we found that SMC1 expression in TNBC tissues exceeded its expression in adjacent non-tumor tissues. Similarly, the expression of SMC1 in TNBC cell lines (hs578T and HCC1937) was found to be higher than in MCF10a and MCF7 cells. Subsequently, SMC1 was overexpressed and silenced in hs578T and HCC1937 cells through plasmid and siRNA transfection, respectively. The results showed that the high expression of SMC1 often promoted EMT, accompanied by the enhanced expression of Brachyury. Besides, upregulated expression of Brachyury through plasmid transfection also significantly improved the level of EMT, which further indicated that SMC1 increased EMT in TNBC through the induction of Brachyury expression. Taken together, these results contributed to a better understanding of the pathogenesis of TNBC, which also provided an experimental basis for the prevention, diagnosis and treatment of TNBC.
\end{abstract}

Correspondence to: Dr Yajie Wang, Department of Oncology, Changhai Hospital Affiliated to The Second Military Medical University, 168 Changhai Road, Yangpu, Shanghai 200433, P.R. China E-mail: yajiewangyjw@163.com

Abbreviations: SMC1, structural maintenance of chromosome 1; EMT, epithelial-mesenchymal transition; TNBC, triple-negative breast cancer

Key words: structural maintenance of chromosome 1, epithelialmesenchymal transition, triple-negative breast cancer, brachyury

\section{Introduction}

Triple-negative breast cancer (TNBC) is a subtype of breast cancer, taking up $15-20 \%$ of all breast cancer cases, which is characterized by more aggressiveness and high rate of proliferation, metastasis, often leading to a poor prognosis and much lower overall survival compared with other subtypes of breast cancer (1-3). Due to its triple-negative expression of estrogen receptor (ER), progesterone receptor (PR) and human epithelial growth factor receptor 2 (HER2), which are the currently available therapeutic targets, TNBC remains an important clinical challenge $(4,5)$. So far, the common therapeutic method for TNBC has been limited to cytotoxic chemotherapy. Hence, there is still a lack of full understanding towards the molecular mechanism of TNBC development.

Cohesin, a highly evolutionary conserved multifunctional nuclear protein complex, is worth noting. Cohesin mainly participates in sister chromatid cohesion, which is also involved in DNA repair (6). Moreover, cohesin has also been shown to be involved in regulation of transcription and cell proliferation, and the maintenance of pluripotency $(7,8)$. Cohesin is composed of two structural maintenance of chromosomes proteins, structural maintenance of chromosomes 1 (SMC1) and structural maintenance of chromosomes 3 (SMC3), and a kleisin protein like RAD21 (6). More specifically, together with SMC3, SMC1 forms an affinitive heterodimer and associates with SCC1/RAD21 and SCC3/SA to form the cohesin complex (9). Among them, SMC1 is known for its role in cell division, DNA repair and activation of the cell cycle checkpoints (10-12). In particular, SMC1 has been shown to contribute to tumor genomic instability, and ectopic expression of cohesin subunits, including SMC1, has been found in sarcoma, melanoma, colon and glioblastoma tumors $(13,14)$. To date, SMC1 has also been reported to be overexpressed in TNBC (15), but the role of SMC1 in the progression of TNBC is not fully understood.

Brachyury is a T-box transcription factor, which plays an important role in the development of vertebrates, including the formation of cervical vertebra, differentiation of the posterior mesoderm and axial development (16). In humans, T-box transcription factors mainly participate in regulating the progenitors and their differentiated descendants (17). Brachyury was also found to be overexpressed in various human malignant neoplasms $(18,19)$, which often contribute 
to the metastasis of tumor cells (20). It is also worth noting that Brachyury could induce epithelial-mesenchymal transition (EMT) in human epithelial cells through the induction of transcription factors, including Snail and Slug (21). However, the role of Brachyury in TNBC and its interactions with EMT remain poorly elucidated.

EMT refers to a series of events that converts epithelial cells into individual migratory cells, during which cells lose epithelial characteristics, such as cell-to-cell adhesion and cell-layer organization, along with acquiring mesenchymal properties including metastasis and invasiveness $(22,23)$. Also, during the EMT process, cells lose epithelial markers, such as E-cadherin, and gain expression of mesenchymal markers, including $\mathrm{N}$-catenin and vimentin $(24,25)$. Hence, EMT has been demonstrated to be the central mechanism responsible for the metastasis and invasiveness of various cancers $(26,27)$. Moreover, EMT is also involved in early embryo development, wound healing, and tissue regeneration (28). However, few studies have focused on its roles in TNBC progression, and no studies have reported its association with SMC1 and Brachyury until now.

In the present study, we compared the expression of SMC1 in TNBC and normal tissues and cells. Subsequently, SMC1 was artificially overexpressed and silenced in TNBC cells. We further analyzed the relationship between SMC1 and EMT, SMC1 and Brachyury, respectively, in TNBC. The upregulation of Brachyury was also investigated to evaluate its role in EMT.

\section{Materials and methods}

Sample collection. In total $40 \mathrm{TNBC}$ and 38 adjacent non-tumor tissue samples were acquired from the Department of Oncology, Xijing Hospital (Xi'an, China). The tissue samples were derived from patients who had not been subjected to preoperative chemotherapy or radiotherapy. The samples were immediately frozen in liquid nitrogen after dissection in preparation for use. All patients with TNBC gave written informed consent for the use of clinical specimens for medical research, and experiments were approved by the Committees for Ethical Review of Research involving human subjects of the Fourth Military Medical University (Xi'an, China).

Cell culture. Two TNBC cell lines (hs578T and HCC1937), a human non-tumorigenic mammary epithelial cell line (MCF10a) and an $\mathrm{ER}^{+} /$hormone responsive breast cancer cell line (MCF7) were acquired from the American Type Culture Collection (ATCC; Manassas, VA, USA). All cells were incubated at $37^{\circ} \mathrm{C}$ in a humidified atmosphere of $5 \% \mathrm{CO}_{2}$ in the appropriate medium supplemented with $10 \%$ fetal bovine serum (FBS); Dulbecco's modified Eagle's medium (DMEM) for hs578T and HCC1937; DMEM/F12 for MCF10a; and RPMI-1640 (all from Invitrogen, Carlsbad, CA, USA) for MCF7.

Plasmid construction and transfection for SMCl and Brachyury overexpression. Human Brachyury and SMC1 were amplified by PCR using cDNA from MCF7. Subsequently, cDNA was sub-cloned into eukaryotic expression vector pcDNA3.1 (Invitrogen). Next, hs578T and HCC1937 cells were transiently transfected with the eukaryotic expression vector (pcDNA3.1) alone or with pcDNA3.1/SMCl and pcDNA3.1/Brachyury, respectively, using Lipofectamine 2000 (Invitrogen) according to the manufacturer's instructions. The stable expression of SMC1 and Brachyury was assessed using real-time quantitative polymerase chain reaction (RT-qPCR) and western blot analysis.

Small interfering RNA (siRNA) transfection for SMC1 silence. The siRNA for SMC1, in addition to non-targeting siRNA, was purchased from GenePharma Co., Ltd. (Shanghai, China). A scrambled siRNA also purchased from GenePharma Co., Ltd., was used as a negative control. Subsequently, siRNA (100 nM) was transfected into hs578T and HCC1937 cells using Lipofectamine 2000 (Invitrogen) for an incubation of $48 \mathrm{~h}$ in antibiotic-free medium. The absent expression of SMC1 was confirmed by RT-qPCR and western blot analysis.

Evaluation of the migratory ability. Cell migration was investigated using Transwell chambers. Briefly, medium supplemented with $10 \%$ FBS was added to the lower chambers, and $1 \times 10^{4}$ cells in serum-free medium were added to the upper chambers. Subsequently, chambers were incubated for $24 \mathrm{~h}$ at $37^{\circ} \mathrm{C}$. The non-migrating cells from the interior of the inserts were gently removed using a cotton-tipped swab. Next, cells on the bottom side of the filters were fixed with methanol and stained with hematoxylin. The migratory ability was determined by counting migratory cells in 5 randomly selected fields under a microscope (Olympus, Tokyo, Japan). Experiments were performed in triplicate.

Evaluation of the proliferative ability. Cell proliferation was evaluated using the BrdU assay. Briefly, prepared BrdU was added into medium, and the mixtures were incubated in 5\% $\mathrm{CO}_{2}$ at $37^{\circ} \mathrm{C}$ for $1 \mathrm{~h}$. Subsequently, cells were fixed with $70 \%$ ethanol and incubated with primary anti-BrdU antibody. Next, cells were counterstained with hematoxylin and counted in randomly selected fields using fluorescence microscopy (Olympus). The labeling index was calculated as BrdU-positive cells vs. total cells.

$R T$ - $q P C R$. Total RNA from tissue samples and cells was extracted using TRIzol reagent (Invitrogen), which was subsequently quantified according to absorbance at $260 \mathrm{~nm}$ using a NanoDrop spectrophotometer (Thermo Fisher Scientific, Waltham, MA, USA). cDNA was synthesized using HiScript ${ }^{\circledR}$ First Strand cDNA Synthesis kit (Vazyme Biotech Co., Ltd., Nanjing, China) and RT-qPCR was performed using HiScript ${ }^{\circledR}$ II Q RT SuperMix for qPCR (Vazyme Biotech Co., Ltd) according to the manufacturer's instructions. The cycle threshold $(\mathrm{Ct})$ was recorded and the fold induction was calculated using the $2^{-\Delta \Delta \mathrm{Ct}}$ method. Each gene was detected in triplicate. GAPDH served as a control. The primer sequences of this experiment are shown in Table I.

Western blotting. Total proteins from tissue samples and cells were extracted in RIPA Lysis and Extraction Buffer (Thermo Fisher Scientific) and quantified using the Bradford method. A total of $50 \mu \mathrm{g}$ of protein was isolated by $10 \%$ sodium dodecyl sulfate-polyacrylamide gel electrophoresis 
Table I. The primer sequences used in this study.

\begin{tabular}{lll}
\hline Gene & \multicolumn{1}{c}{ Sense } & \multicolumn{1}{c}{ Antisense } \\
\hline SMC1 & 5'-GGCGGATCCATGGTTCCTGAATGAT-3' & 5'-CCGCTCGAGCTACTGCTCATTGGGGTT-3' \\
Brachyury & 5'-ACTGAGAATCAGCCGGACTT-3' & 5'-CTGCACTGCAAAGAACCACT-3' \\
E-cadherin & 5'-TTAAACTCCTGGCCTCAAGCAATC-3' & 5'-TCCTATCTTGGGCAAAGCAACTG-3' \\
N-cadherin & 5'-CTCCTATGAGTGGAACAGGAACG-3' & 5'-TTGGATCAGTCATAATCAAGTGCTGTA-3' \\
Vimentin & 5'-ATGTGGATGTTTCCAAGCCTGAC-3' & 5'-GAGTGGGTATCAACCAGAGGGAG-3' \\
Snail & 5'-TTCTTCGCTACTGCTGCG-3' & 5'-GGGCAGGTATGGAGAGGAAGA-3' \\
Slug & 5'-ATCTGACCCGTCGTGACG-3' & 5'-CGTCACGACGGGTCAGAT-3' \\
GAPDH & 5'-AGGTCGGTGTGAACGGATTTG-3' & 5'-GGGGTCGTTGATGGCAACA-3' \\
\hline
\end{tabular}

Table II. The primary antibodies used in this study.

\begin{tabular}{llllc}
\hline Protein & Host & Cat no. & Commercial source & Dilutions \\
\hline SMC1 & Goat & sc-21078 & Santa Cruz Biotechnology & $1: 1,000$ \\
Brachyury & Goat & sc-17743 & Santa Cruz Biotechnology & $1: 1,000$ \\
E-cadherin & Rabbit & sc-7870 & Santa Cruz Biotechnology & $1: 1,000$ \\
N-cadherin & Rabbit & sc-7939 & Santa Cruz Biotechnology & $1: 1,000$ \\
Vimentin & Goat & sc-7557 & Santa Cruz Biotechnology & $1: 1,500$ \\
Snail & Goat & sc-10433 & Santa Cruz Biotechnology & $1: 500$ \\
Slug & Goat & sc-10436 & Santa Cruz Biotechnology & $1: 500$ \\
GAPDH & Goat & sc-20357 & Santa Cruz Biotechnology & $1: 3,000$ \\
\hline
\end{tabular}

A

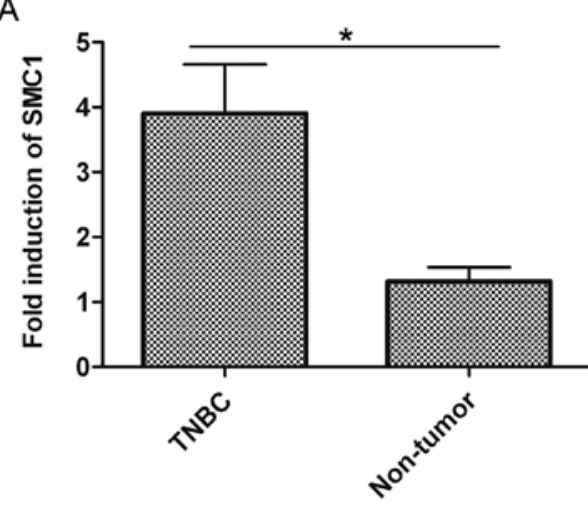

B

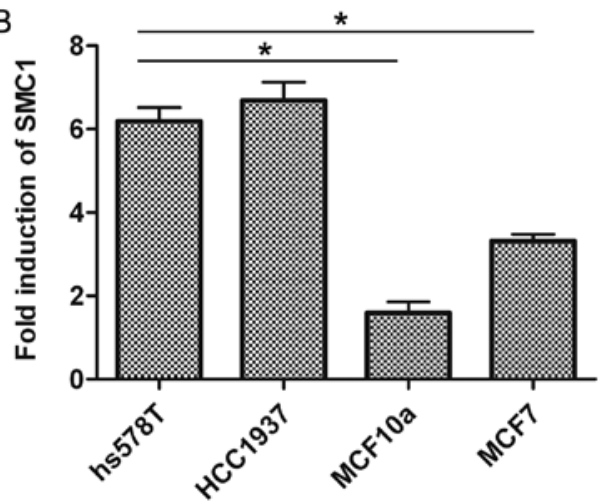

Figure 1. The expression of SMC1 in TNBC tissues and cells. RT-qPCR was performed. (A) SMC1 expression in TNBC tissues (n=40) was higher than in adjacent non-tumor tissues (n=38). (B) TNBC cells (hs578T and HCC1937) showed higher expression of SMC1 compared with MCF10a and MCF7 cells. Values were presented as mean $\pm \mathrm{SD}$; each experiment was performed in triplicate; ${ }^{*} \mathrm{P}<0.05$.

(SDS-PAGE), followed by electro-blotting onto polyvinylidene fluoride (PVDF) membranes (Millipore, Billerica, MA, USA). Subsequently, the PVDF membranes were incubated overnight at $4^{\circ} \mathrm{C}$ with primary antibodies (as shown in Table II). After incubation with horseradish peroxidase (HRP)-conjugated secondary antibodies (Santa Cruz Biotechnology, Santa Cruz, CA, USA) at $37^{\circ} \mathrm{C}$ for $2 \mathrm{~h}$, bound proteins were visualized by 4-chloro-1-naphthol (4-CN) and detected by chemiluminescence (ECL) detection system (Amersham, Little Chalfont, UK).

Statistical analysis. Statistical analysis was performed using SPSS 19.0 (SPSS Inc., Chicago, IL, USA). Data were presented as mean \pm standard deviation (SD). The statistical differences between two groups were determined by Student's t-test. A p-value $<0.05$ was considered statistically significant.

\section{Results}

SMC1 is highly expressed in TNBC tissues and cells. Firstly, we compared SMC1 expression in TNBC with its expression in non-TNBC conditions. RT-qPCR was performed. As shown in Fig. 1A, compared with adjacent non-tumor tissues, SMC1 expression in TNBC tissues was significantly higher. Also, the expression of SMC1 in two TNBC cell lines, hs578T 
A

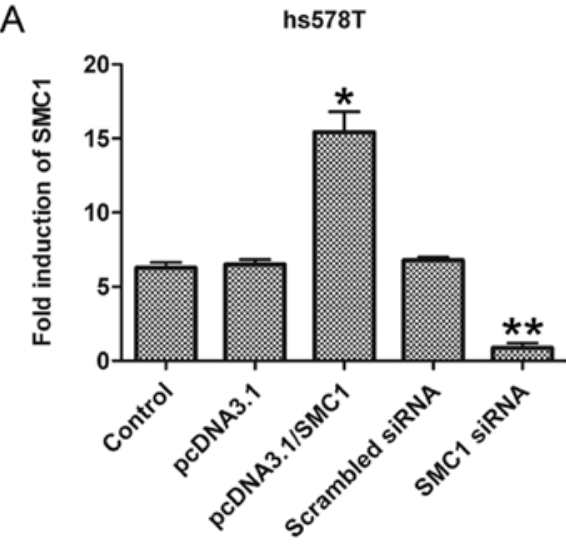

C

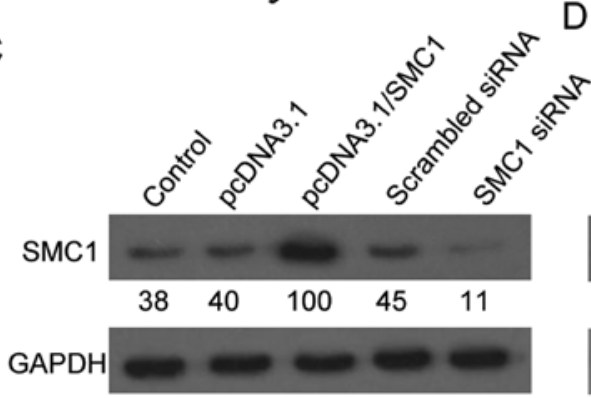

$\mathrm{B}$

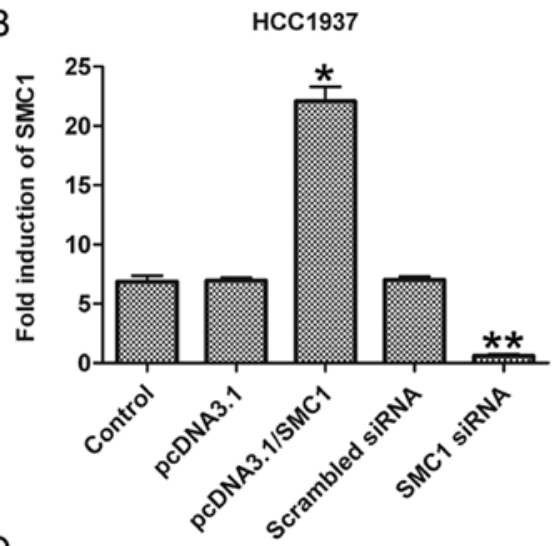

D

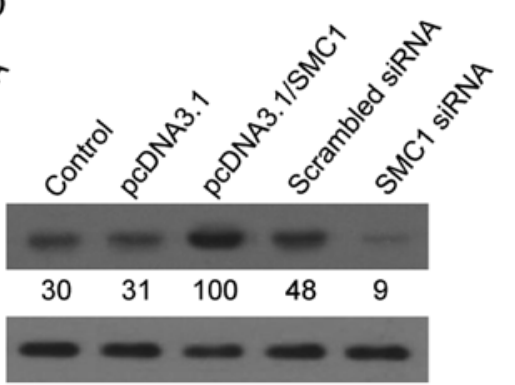

Figure 2. The overexpression and silence of SMC1, respectively, in both hs578T and HCC1937. RT-qPCR and western blotting were performed. In hs578T cells, the expression of SMC1 was upregulated by pcDNA3.1/SMC1 transfection, but downregulated by SMC1 siRNA transfection in mRNA level (A) and protein level (C). (B and D) Similar alterations were observed in HCC1937 cells. Values were presented as mean \pm SD; each experiment was performed in triplicate; ${ }^{*} \mathrm{P}<0.05$ and ${ }^{* *} \mathrm{P}<0.01$ vs. control group.

and HCC1937, was found to exceed its expression in normal mammary epithelial cells (MCF10a) and non-TNBC breast cancer cells (MCF7) (Fig. 1B). These results suggested that SMC1 was abnormally elevated in TNBC nidus.

SMC1 is overexpressed after plasmid transfection, but silenced by siRNA transfection. Next, to further investigate the effects of SMC1 on TNBC progression, SMC1 was artificially overexpressed and silenced through plasmid and siRNA transfection, respectively, in hs578T and HCC1937 cells. As shown in Fig. 2A, SMC1 showed remarkably upregulated expression of 15.4 \pm 1.38 -fold after pcDNA3.1/SMCl transfection, and markedly downregulated expression of $0.88 \pm 0.3$-fold after SMC1 siRNA transfection in hs578T cells, compared with the control group; similarly, in HCC1937 cells, pcDNA3.1/SMCl group had a significantly improved expression of SMC1 of 22.1 1.23 fold, and SMC1 siRNA group with an inhibited expression of $0.6 \pm 0.15$-fold (Fig. 2B). The above results were also confirmed by western blotting at the protein level (Fig. 2C and D). These results demonstrated the successful regulation of SMC1 expression via exogenous transfection.

SMC1 overexpression promotes EMT process. As EMT has been widely accepted as a major process participating in cancer development, we next explored the role of SMC1 in regulating EMT in TNBC cells. Upregulation of SMC1 in both hs578T and HCC1937 cells led to decreased expression of the epithelial marker E-cadherin (Fig. 3A) and increased expression of mesenchymal markers, such as N-cadherin, vimentin, Snail and Slug (Fig. 3B-E). Western blotting results also revealed that the protein levels of $\mathrm{N}$-cadherin, vimentin, Snail and Slug were promoted, whereas E-cadherin was inhibited in SMC1-overexpressing cells compared with the control group (Fig. 3F and G). On the contrary, downregulation of SMC1 resulted in relatively stable expression of E-cadherin, $\mathrm{N}$-cadherin, vimentin, Snail and Slug, without statistical significance compared with the control group (Fig. 3A-E). These results indicated that high expression of SMC1 promoted the EMT process.

SMC1 overexpression promotes metastasis and proliferation of TNBC cells. As EMT has also been reported to be closely correlated with the survival of tumor cells $(20,29)$, we next aimed to evaluate the effects of SMC1 overexpression on the metastasis and proliferation of TNBC cells. As a result, after SMC1 overexpression, the average number of migratory cells was $73 \pm 4.8$ in hs578T and $84 \pm 4.2$ in HCC1937, which was significantly higher than that of the control group $(25 \pm 2.5$ and 31 2.0 , respectively; Fig. 4A). In contrast, the results also showed that after SMC1 knockdown, the average number of migratory cells $(10 \pm 3.6$ in hs578T and $15 \pm 2.5$ in HCC1937) was significantly reduced compared with the control group (Fig. 4A). The proliferative ability was also enhanced by SMC1 overexpression, but inhibited in SMC1 silencing cells (Fig. 4B). These data further supported the involvement of SMC1 in EMT as observed in TNBC cells.

Brachyury expression is also elevated in TNBC tissues and cells. This study aimed to introduce Brachyury and reveal its relationship with TNBC. Fig. 5A shows that Brachyury was 
A

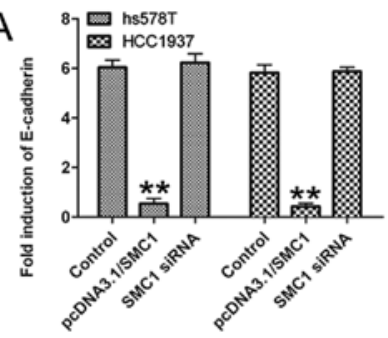

B
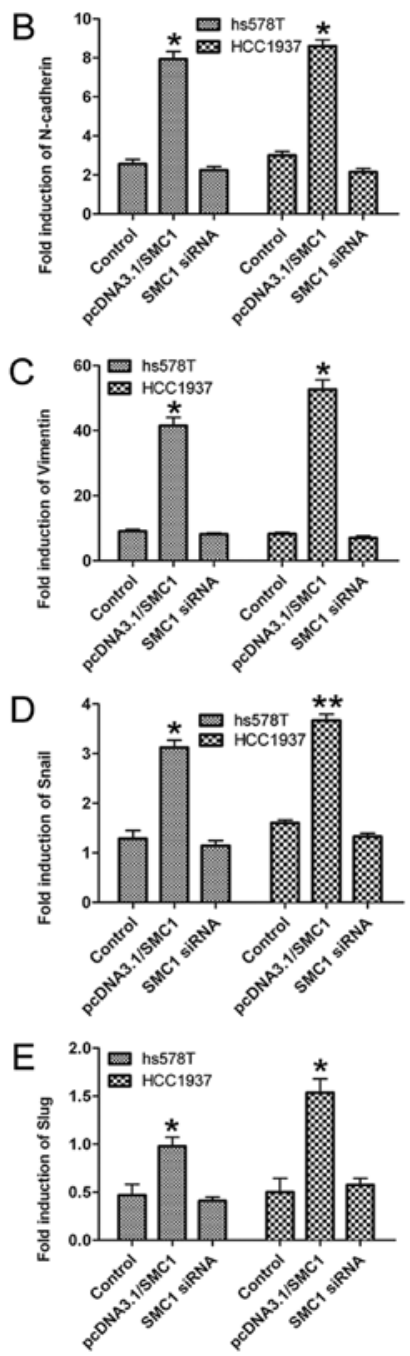
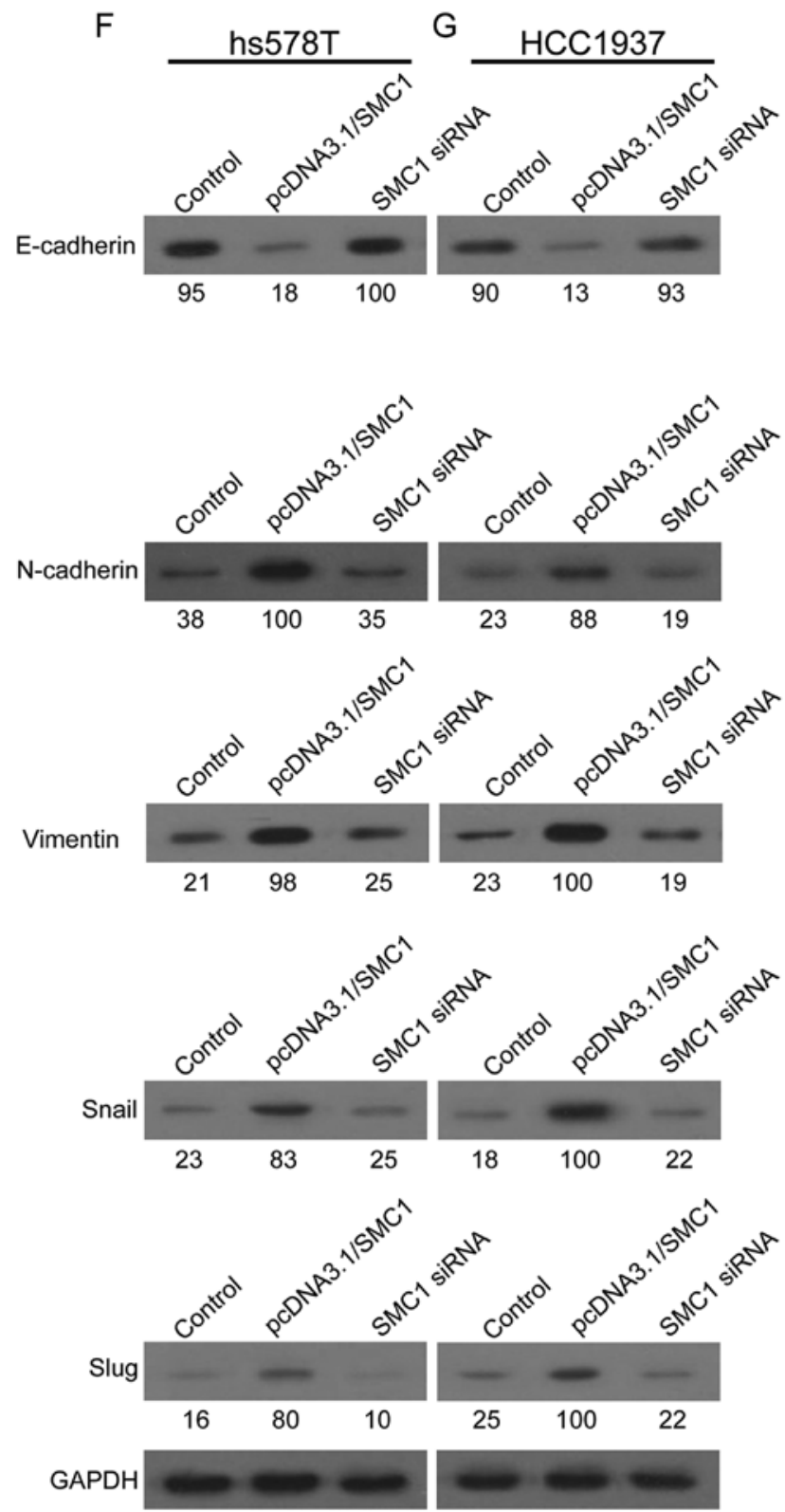

Figure 3. The variations of the expression of EMT markers caused by SMC1. RT-qPCR and western blotting were performed. (A) E-cadherin expression was decreased by SMC1 overexpression, but slightly increased by SMC1 knockdown. The expression of mesenchymal markers, including N-cadherin (B), vimentin (C), Snail (D) and Slug (E), was upregulated by SMC1 overexpression, whereas remained constant compared with control group. The protein expression in hs578T (F) and HCC1937 (G) cells was also confirmed by western blotting results. Values were presented as mean \pm SD; each experiment was performed in triplicate; $\mathrm{P}<0.05$ and ${ }^{* *} \mathrm{P}<0.01$ vs. control group.

strongly expressed in TNBC tissues. There was also a higher expression of Brachyury in TNBC cells than in MCF10a and MCF7 cells (Fig. 5B), implying that TNBC was accompanied by the high expression of Brachyury.

Overexpression of SMC1 indicates ectopic expression of Brachyury. Since SMC1 and Brachyury were both highly expressed in TNBC tissues and cells, the present study aimed to uncover the association between SMC1 and Brachyury. As shown in Fig. 6, high expression of SMC1 usually upregulated the expression of Brachyury; conversely, knockdown of SMC1 led to decreased expression of Brachyury, at both the mRNA and protein level. These results indicated that Brachyury may be a downstream effector of the SMC1 gene.

Overexpression of Brachyury promotes the EMT process. Brachyury has previously been reported to induce the EMT process in human epithelial cells; hence, we further investigated whether SMC1 promoted EMT through the induction of Brachyury expression in TNBC cells. Brachyury was overexpressed through plasmid transfection in hs578T and HCC1937 cells (Fig. 7). Brachyury overexpression markedly inhibited E-cadherin expression (Fig. 8A), while it remarkably promoted the expression of the two mesenchymal markers 
A

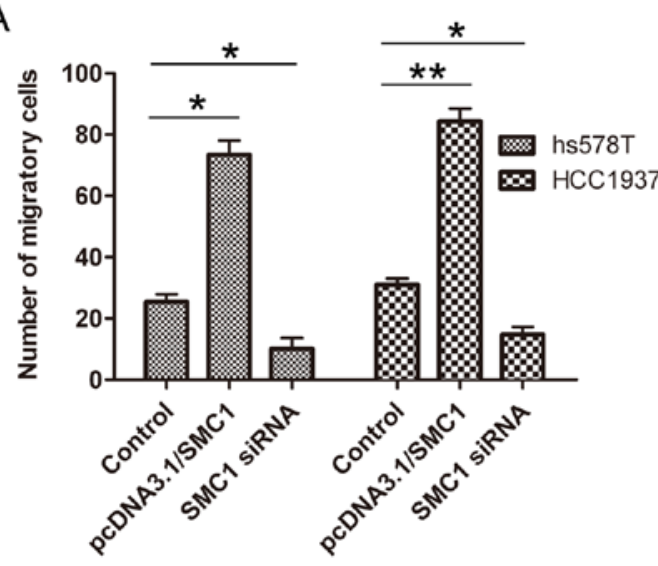

B

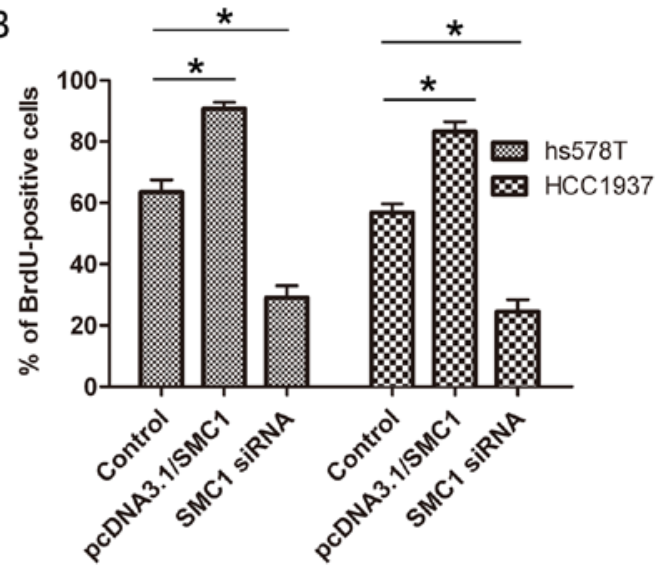

Figure 4. The effects of SMC1 on metastasis and proliferation of TNBC cells. Transwell assay and BrdU assay were performed. (A) SMC1 overexpression promoted, but SMC1 knockdown inhibited the metastasis of hs578T and HCC1937 cells. (B) The proliferation was also increased by SMC overexpression, but decreased by silence of SMC1. Values are presented as mean $\pm \mathrm{SD}$; each experiment was performed in triplicate; ${ }^{*} \mathrm{P}<0.05$ and ${ }^{* *} \mathrm{P}<0.01 \mathrm{vs}$. control group.
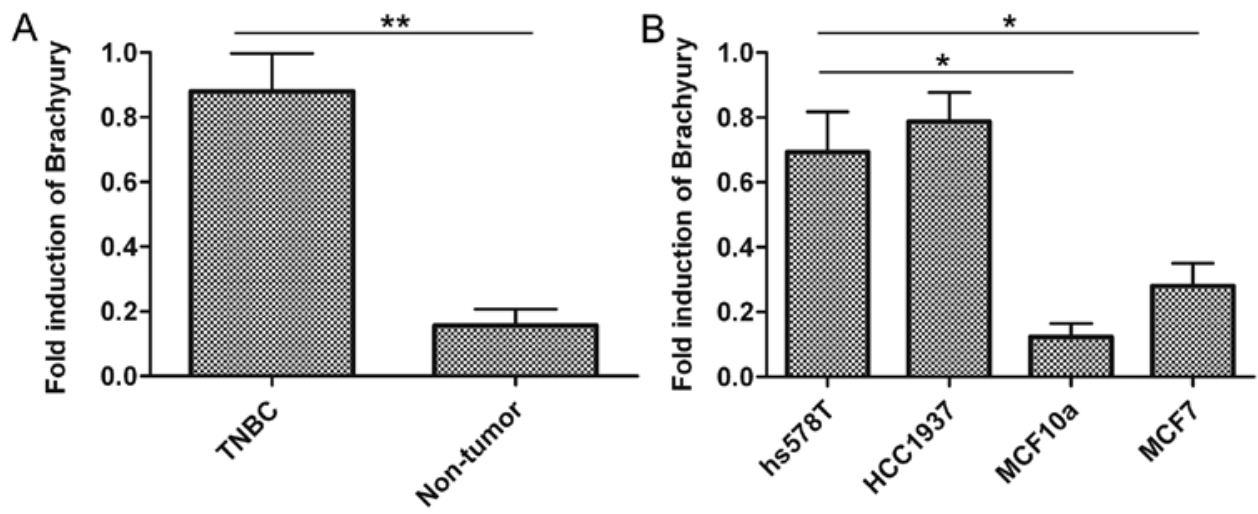

Figure 5. The expression of Brachyury in TNBC tissues and cells. RT-qPCR was performed. Brachyury was highly expressed in TNBC tissues (A) and cells (B). Values are presented as mean $\pm \mathrm{SD}$; each experiment was performed in triplicate; ${ }^{*} \mathrm{P}<0.05$ and ${ }^{* * *} \mathrm{P}<0.01$.

A

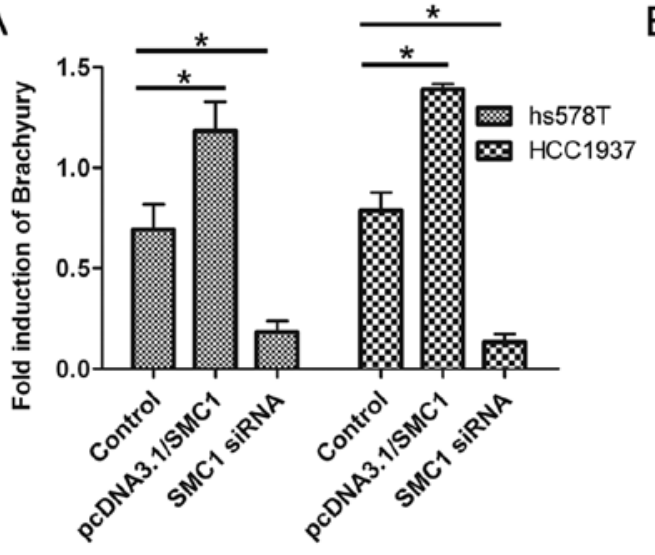

B

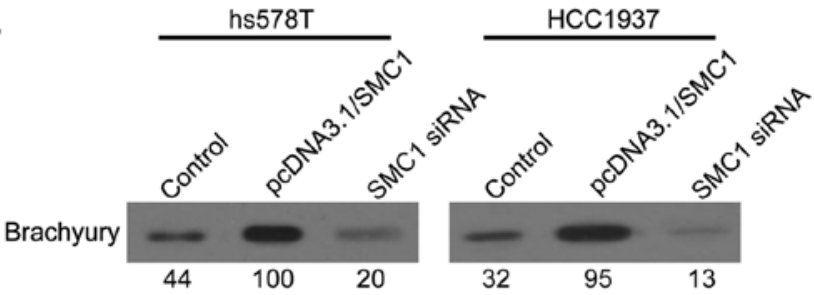

GAPDH

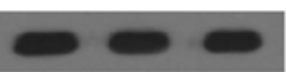

Figure 6. The effects of SMC1 on the expression of Brachyury in TNBC cells. RT-qPCR and western blotting were performed. (A) Brachyury expression in mRNA level was increased by SMC1 overexpression, but decreased by SMC1 knockdown in both hs578T and HCC1937 cells. (B) The western blotting also showed the upregulated expression of Brachyury in the presence of pcDNA3.1/SMC1. Values were presented as mean \pm SD; each experiment was performed in triplicate; ${ }^{*} \mathrm{P}<0.05$ vs. control group.

N-cadherin (Fig. 8B) and vimentin (Fig. 8C), indicating that Brachyury was able to promote the EMT process. These data further demonstrated that SMC1 increased EMT in TNBC, possibly through the induction of Brachyury expression.

\section{Discussion}

In the present study, we explored the expression of SMC1 in TNBC; correspondingly, SMC1 was found to be highly 

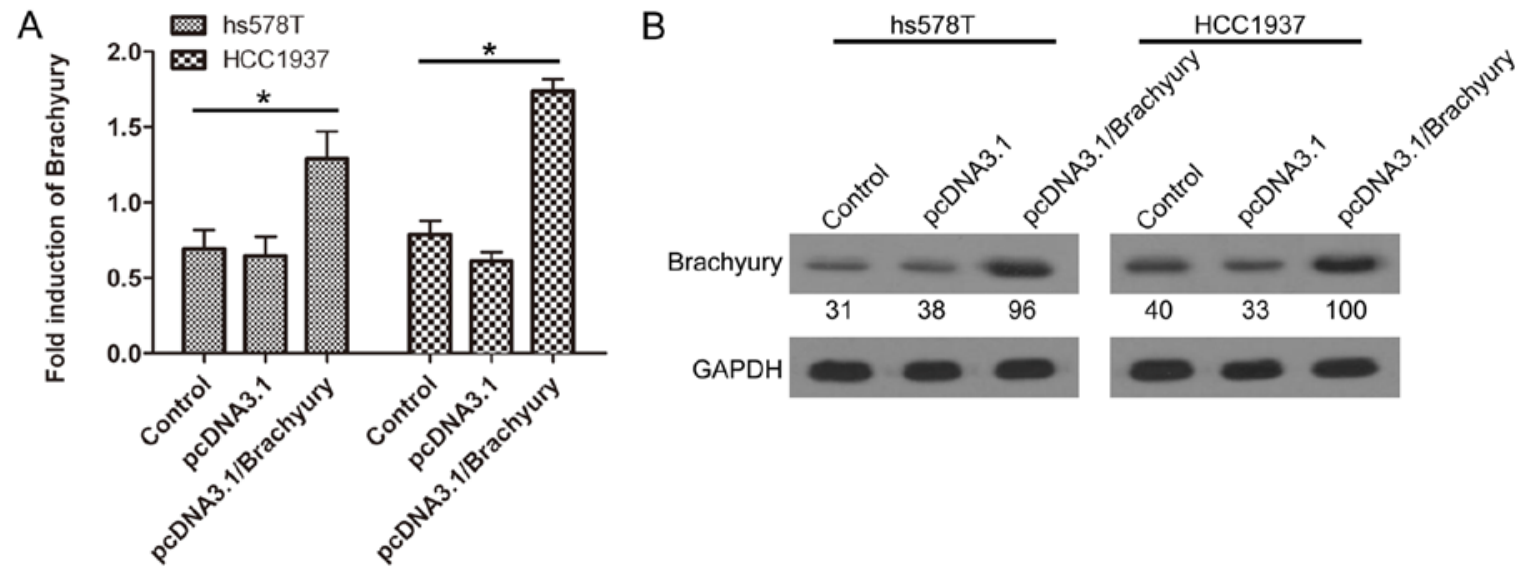

Figure 7. The overexpression of Brachyury through plasmid transfection in TNBC cells. RT-qPCR and western blotting were performed. Brachyury was overexpressed in hs578T and HCC1937 cells in mRNA (A) and protein (B) levels. Values are presented as mean \pm SD; each experiment was performed in triplicate; ${ }^{*} \mathrm{P}<0.05$ vs. control group.

A

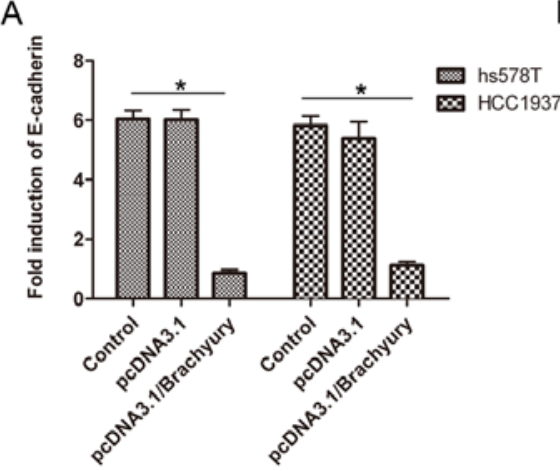

B

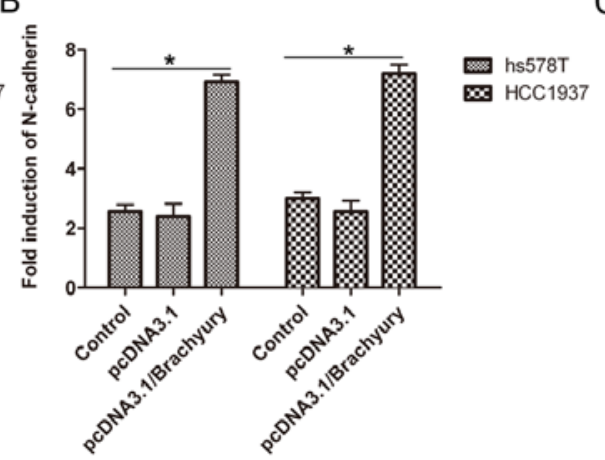

C

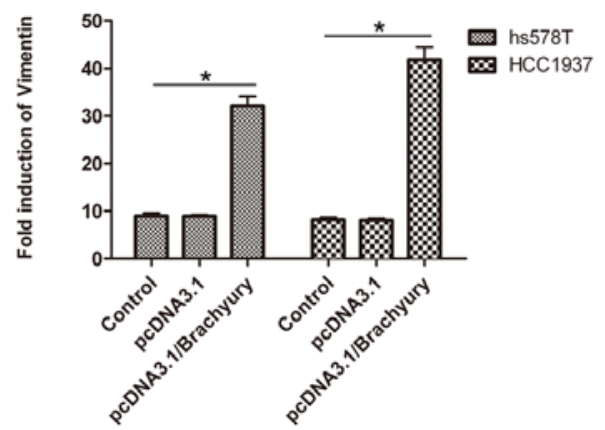

Figure 8. The effects of Brachyury overexpression on EMT markers. RT-qPCR was performed. (A) E-cadherin expression was significantly downregulated by SMC1 overexpression. The expression of N-cadherin (B) and vimentin (C) was markedly upregulated after pcDNA3.1/SMC1 transfection. Values are presented as mean $\pm \mathrm{SD}$; each experiment was performed in triplicate; ${ }^{*} \mathrm{P}<0.05$ vs. control group.

expressed in both TNBC tissues and cells. To further investigate the effects of SMC1 on TNBC, SMC1 was overexpressed and silenced in hs578T and HCC1937 cells, respectively. Interestingly, SMC1 overexpression was found to be able to induce the EMT process in TNBC cells. Also, SMC1 was able to promote the metastasis and proliferation of TNBC cells. Brachyury was also found to be involved in this study. The results showed that Brachyury may be a downstream molecule of SMC1, participating in induction of the EMT process. The high expression of SMC1 was accompanied by the upregulated expression of Brachyury. These consistent clues could provide a potential target for the diagnosis and treatment of TNBC.

As described, the increased expression of SMC1 in TNBC was confirmed in this study. Yadav et al also focused on the study of SMC1 and TNBC. They found that SMC1 played a role in cell migration and drug sensitivity of TNBC cells. At the same time, they declared that they had discovered the overexpression of SMC1 in TNBC for the first time (15). Our results show for the first time that the ectopic expression of SMC1 increased metastasis and the proliferation of TNBC cells, possibly through induction of the EMT process.

TNBC is a malignant neoplasm, which is characterized by high capability of metastasis and invasiveness (15), thus, EMT was introduced to this study due to its profound involvement in promoting metastasis and invasiveness (30). Briefly, during the EMT process, cells may lose epithelial markers and adherent ability, and gain mesenchymal markers and invasive capability, which can convert normal cells into tumor cells, contributing to the metastasis and invasiveness of the tumor (29). Hence, the relationship between EMT and TNBC needs to be urgently investigated. In the present study, the data suggested that SMC1 promoted metastasis and proliferation of TNBC cells through regulation of its EMT phenotype. This notion was based on the following findings: i), mesenchymal markers were significantly upregulated in the SMC1-overexpressed TNBC cell lines, whereas the epithelial markers were remarkably decreased; SMC1 knockdown showed the opposite effects; ii), western blotting results also confirmed these alterations; and iii), Transwell and BrdU assays demonstrated that enforced SMC1 led to increased metastasis and proliferation, whereas decreased SMC1 expression resulted in inhibited metastasis and proliferation of TNBC cells. Therefore, these data indicated that SMC1 promoted TNBC metastasis and proliferation, possibly through the induction of EMT in TNBC cells.

Next, Brachyury was introduced into this experiment. A previous study reported that Brachyury was highly expressed in human tumor cells, but not in the corresponding normal 
cell lines (29). Fernando et al reported that Brachyury could induce EMT in human epithelial cells through the repression of E-cadherin and the induction of Slug (31). Moreover, Brachyury was also reported to promote EMT in hepatocellular carcinoma (20), oral squamous cell carcinoma (29) and non-small cell lung cancer (32). In accordance with the above results, the present study showed that Brachyury was positively expressed in TNBC tissues and cells, whereas it was negatively expressed in normal tissues and cells. We subsequently aimed to investigate whether Brachyury participated in the SMC1-induced EMT in TNBC cells. The expression of Brachyury was found to be upregulated along with SMC1 overexpression, indicating that Brachyury may be an effector of SMC1 in TNBC cells. Besides, overexpression of Brachyury in TNBC cells also induced characteristic changes of EMT, including elevated levels of mesenchymal markers and decreased levels of epithelial markers. Based on these data, we suggested that Brachyury expression was linked to EMT and SMC1 expression in TNBC cells.

In conclusion, the present study demonstrated that SMC1 could promote the EMT process through the induction of Brachyury expression in TNBC. SMC1, in combination with Brachyury, could serve as a novel target for the prevention and treatment of TNBC, which will shed new light on understanding TNBC progression and metastasis.

\section{Acknowledgements}

The present study was supported by the National Natural Science Foundation of China (no. 81072175 and 81372854).

\section{References}

1. Ossovskaya V, Wang Y, Budoff A, Xu Q, Lituev A, Potapova O, Vansant G, Monforte J and Daraselia N: Exploring molecular pathways of triple-negative breast cancer. Genes Cancer 2: 870-879, 2011

2. Metzger-Filho O, Tutt A, de Azambuja E, Saini KS, Viale G,Loi S, Bradbury I, Bliss JM, Azim HA Jr, Ellis P, et al: Dissecting the heterogeneity of triple-negative breast cancer. J Clin Oncol 30: 1879-1887, 2012

3. Liu H, Scholz C, Zang C, Schefe JH, Habbel P, Regierer AC, Schulz CO, Possinger K and Eucker J: Metformin and the mTOR inhibitor everolimus (RAD001) sensitize breast cancer cells to the cytotoxic effect of chemotherapeutic drugs in vitro. Anticancer Res 32: 1627-1637, 2012.

4. Stebbing J and Ellis P: An overview of drug development for metastatic breast cancer. Br J Nurs 21: S18-S22, 2012.

5. Brouckaert O, Wildiers H, Floris G and Neven P: Update on triple-negative breast cancer: Prognosis and management strategies. Int J Womens Health 4: 511-520, 2012.

6. Laugsch M, Seebach J, Schnittler H and Jessberger R: Imbalance of SMC1 and SMC 3 cohesins causes specific and distinct effects. PLoS One 8: e65149, 2013.

7. Liu Z, Scannell DR, Eisen MB and Tjian R: Control of embryonic stem cell lineage commitment by core promoter factor, TAF3. Cell 146: 720-731, 2011

8. Rhodes JM, McEwan M and Horsfield JA: Gene regulation by cohesin in cancer: Is the ring an unexpected party to proliferation? Mol Cancer Res 9: 1587-1607, 2011.

9. Nasmyth $\mathrm{K}$ and Haering $\mathrm{CH}$ : The structure and function of SMC and Kleisin complexes. Annu Rev Biochem 74: 595-648, 2005.

10. Yazdi PT, Wang Y, Zhao S, Patel N, Lee EY and Qin J: SMC1 is a downstream effector in the ATM/NBS1 branch of the human S-phase checkpoint. Genes Dev 16: 571-582, 2002.
11. Michaelis C, Ciosk R and Nasmyth K: Cohesins: Chromosomal proteins that prevent premature separation of sister chromatids. Cell 91: 35-45, 1997.

12. Hirano T: At the heart of the chromosome: SMC proteins in action. Nat Rev Mol Cell Biol 7: 311-322, 2006.

13. Rocquain J, Gelsi-Boyer V, Adélaïde J, Murati A, Carbuccia N, Vey N, Birnbaum D, Mozziconacci MJ and Chaffanet M: Alteration of cohesin genes in myeloid diseases. Am J Hematol 85: 717-719, 2010.

14. Yamamoto G, Irie T, Aida T, Nagoshi Y, Tsuchiya R and Tachikawa T: Correlation of invasion and metastasis of cancer cells, and expression of the RAD21 gene in oral squamous cell carcinoma. Virchows Arch 448: 435-441, 2006.

15. Yadav S, Sehrawat A, Eroglu Z, Somlo G, Hickey R, Yadav S, Liu X, Awasthi YC and Awasthi S: Role of SMC1 in overcoming drug resistance in triple negative breast cancer. PLoS One 8: e64338, 2013

16. Naiche LA, Harrelson Z, Kelly RG and Papaioannou VE: T-box genes in vertebrate development. Annu Rev Genet 39: 219-239, 2005.

17. Showell C, Binder O and Conlon FL: T-box genes in early embryogenesis. Dev Dyn 229: 201-218, 2004.

18. Kilic N, Feldhaus S, Kilic E, Tennstedt P, Wicklein D, Wasielewski R, Viebahn C, Kreipe H and Schumacher U: Brachyury expression predicts poor prognosis at early stages of colorectal cancer. Eur J Cancer 47: 1080-1085, 2011.

19. Yang XR, Ng D, Alcorta DA, Liebsch NJ, Sheridan E, Li S, Goldstein AM, Parry DM and Kelley MJ: T (brachyury) gene duplication confers major susceptibility to familial chordoma. Nat Genet 41: 1176-1178, 2009.

20. Du R, Wu S, Lv X, Fang H, Wu S and Kang J: Overexpression of brachyury contributes to tumor metastasis by inducing epithelial-mesenchymal transition in hepatocellular carcinoma. J Exp Clin Cancer Res 33: 105, 2014.

21. Huang B, Cohen JR, Fernando RI, Hamilton DH, Litzinger MT, Hodge JW and Palena C: The embryonic transcription factor Brachyury blocks cell cycle progression and mediates tumor resistance to conventional antitumor therapies. Cell Death Dis 4: e682, 2013.

22. Thiery JP, Acloque H, Huang RY and Nieto MA: Epithelialmesenchymal transitions in development and disease. Cell 139: 871-890, 2009.

23. Acloque H, Adams MS, Fishwick K, Bronner-Fraser M and Nieto MA: Epithelial-mesenchymal transitions: The importance of changing cell state in development and disease. J Clin Invest 119: 1438-1449, 2009.

24. Thiery JP and Sleeman JP: Complex networks orchestrate epithelial-mesenchymal transitions. Nat Rev Mol Cell Biol 7: 131-142, 2006.

25. Savagner P: Leaving the neighborhood: Molecular mechanisms involved during epithelial-mesenchymal transition. BioEssays 23: 912-923, 2001.

26. Larue L and Bellacosa A: Epithelial-mesenchymal transition in development and cancer: Role of phosphatidylinositol 3' kinase/AKT pathways. Oncogene 24: 7443-7454, 2005.

27. Christofori G: New signals from the invasive front. Nature 441: 444-450, 2006

28. Kalluri R: EMT: When epithelial cells decide to become mesenchymal-like cells. J Clin Invest 119: 1417-1419, 2009.

29. Imajyo I, Sugiura T, Kobayashi Y, Shimoda M,Ishii K, AkimotoN, Yoshihama N, Kobayashi I and Mori Y: T-box transcription factor Brachyury expression is correlated with epithelial-mesenchymal transition and lymph node metastasis in oral squamous cell carcinoma. Int J Oncol 41: 1985-1995, 2012.

30. Lim J and Thiery JP: Epithelial-mesenchymal transitions: Insights from development. Development 139: 3471-3486, 2012.

31. Fernando RI, Litzinger M, Trono P, Hamilton DH, Schlom J and Palena C: The T-box transcription factor Brachyury promotes epithelial-mesenchymal transition in human tumor cells. J Clin Invest 120: 533-544, 2010.

32. Xu K, Liu B and Liu Y: Impact of Brachyury on epithelial-mesenchymal transitions and chemosensitivity in non-small cell lung cancer. Mol Med Rep 12: 995-1001, 2015. 